SINAI Journal of Applied Sciences (ISSN: 2314-6079) Vol. (8) Is. (2), Aug. 2019

scresned BY SINAI Journal of Applied Sciences

\title{
PERFORMANCE OF SOME QUINOA (Chenopoduim quinoa Willd.) GENOTYBES UNDER DIFFERENT LEVELS OF SALINITY
}

\author{
Asmaa M. El-Azraq ${ }^{*}$, D.A. Abd-Moneim and Eman I. El-Sarag
}

1. Dept. Plant Prod., Fac. Environ. Agric. Sic., Arish Univ., Egypt

\begin{abstract}
An experiment was conducted at greenhouse, Faculty of Environmental Agricultural Sciences, Arish University, North Sinai Governorate, Egypt, during two consecutive winter seasons $(2016 / 2017 \& 2017 / 2018)$ to investigate the effect of four levels of salinity (control 60, 80, 120, $160 \mathrm{Mm}$ Sodium Chloride) on five genotypes of quinoa plant (M-28, Q-37, S-10, Regeolone-3, Line-6). Complete randomized design (CRD) was used in three replicates in this experimental work. Petri dishes of three replicate were used to determine the proportion and rate of quinoa germination ability. The results of the experiment indicated that germination rate and germination percentage of quinoa genotypes were significantly affected by salinity levels. The highest germination rate and percentage were $77.27,93.76 \%$ at $2016 / 2017$ and $92.18,93.76 \%$ at $2017 / 2018$ which obtained by M-28 genotype under control treatment (60 $\mathrm{Mm})$ at both seasons. However, the lowest germination rate and percentage were 40.35, $34.73 \%$ at $2016 / 2017$ and $55.27,35.04 \%$ at 2017/2018 which achieved by Line- 6 genotype under $160 \mathrm{Mm}$ treatment at the both seasons. The all studied characters were significantly reduced by increasing the levels of salinity. The characters of grain weight/plant, harvest index and 1000 seeds weight were reduced significantly and gradually by increasing salinity levels from control treatment to $160 \mathrm{Mm}$ treatment.
\end{abstract}

Key words: Chenopoduim quinoa, Germination, Harvest Index (HI\%) and Salinity.

\section{INTRODUCTION}

Quinoa (Chenopoduim. quinoa Willd.) is an allotetra ploid plant species displaying disomic inheritance and belongs to the amaranthaceae family in the subfamily Chenopodioideae, which widely cultivated in South America, mainly in the arid and semiarid areas of the Andean region (Stevens et al., 2006). It has multi economic uses quinoa; highly nutritive values are being used to make flour, soup, breakfast and alcohol, while, quinoa flour, in combination with wheat flour or corn meal is used in making biscuits, bread and processed food. Salinity is one of the most widespread environmental threats to global crop production, especially in arid and semi-arid climates, where land degradation, water shortage and population. Growth is a major concern of salt tolerance in $C$. quinoa, which a prerequisite for its sustainable utilization as non-conventional crop using alternative water sources on marginal lands (Eisa et al., 2012). This crop is well adapted to different environmental conditions, including water scarcity, low temperatures, salinity and poor soils (Bascunan-Godo et al., 2015). So, it has been considered an important crop with the potential of contributing to food security worldwide (FAO, 2011).

Begum et al. (2010) investigated the response of wheat growth to different salinity levels $\left(0.0,4,8,12,16 \mathrm{dsm}^{-1}\right)$. They used 50 grains per petri dish and illustrated that the germination percentage decreased in high salinity level. Also water uptake decreased with an increase of

\footnotetext{
* Corresponding author: Tel.: +20125044129

E-mail address: asmaa.mustafa@yahoo.com
} 
salinity level, but it was not so much drastic up to $8 \mathrm{dsm}^{-1}$. Accumulation of $\mathrm{Na}^{+}$and $\mathrm{CI}^{-}$ increased, when the grains were treated with $4 \mathrm{dsm}^{-1}$ to $16 \mathrm{dsm}^{-1}$ saline solutions.

In quinoa plant, Hirich et al. (2014) evaluated its response to different irrigation water salinity treatments $(1,10,20$ and 30 ds $\left.\mathrm{m}^{-1}\right)$. They showed that increasing salinity affected significantly grain yield, harvest index (HI). The highest HI was obtained under most stressed treatment (30 ds $\mathrm{m}^{-1}$ ), while, the lowest values were obtained under treatment received saline water with an $\mathrm{EC}$ value equal to $10 \mathrm{ds} \mathrm{m}^{-1}$.

Panuccio et al. (2014) evaluated the effect of saline water on seed germination of the halophyte quinoa. Seeds were germinated in Petri dishes with sea water (SW) solutions $(25,50,75$, and $100 \%)$ of $\mathrm{Na} \mathrm{Cl}, \mathrm{Ca} \mathrm{Cl}, \mathrm{KCl}$ and $\mathrm{Mg} \mathrm{Cl}_{2}$ individually, at the concentrations in which they are present in SW. They were found that all salts, at lower concentrations, increased the germination rate, but not the germination percentages, compared with control (pure water). Conversely, seedlings were differently affected by treatments in respect to salt type and concentration.

Also, Algosaibi et al. (2015) studied the effect of four salinity treatments $(1.25,4,8$, $16 \mathrm{ds} \mathrm{m}^{-1}$ ) on grain weigh, 1000-grain weight and dry weight per plant of quinoa.
Results clarified that the low values of grain yield were recorded at $16 \mathrm{ds} \mathrm{m}^{-1}(17.05 \mathrm{~g} /$ plant), while, the highest values were recorded with $4 \mathrm{ds} \mathrm{m}^{-1}$ treatment $(34.08 \mathrm{~g} /$ plant). 1000-grain weight values were ranged between $2.97 \mathrm{~g}$ at $4 \mathrm{ds} \mathrm{m}^{-1}$ treatment, and $3.49 \mathrm{~g}$ at treatment $1.25 \mathrm{ds} \mathrm{m}^{-1}$.

Germination rate in Petri dishes with three replications of ten quinoa cultivars was studied by Tan and kcay (2017). They found that germination rates of quinoa cultivars ranged from 0 to $87.3 \%$ and $100 \%$ was determined at the Q-52 cultivar. As salinity level increases, the germination rates were decreased. Q-52 cultivar had the maximum germination rate $(98 \%)$ in the salt-free conditions.

\section{MATERIALS AND METHODS}

This study was carried out at greenhouse, Faculty of Environmental Agricultural Sciences, Arish University, North Sinai, Governorate, Egypt ( $31^{\circ} 08^{\prime} 40.3 \mathrm{~N}^{\prime \prime}, 33^{\circ}$ $\left.49^{\prime} 37.2^{\prime \prime}\right)$, during two winter successive seasons (2016/ 2017 and 2017/2018), to investigate the effect of four levels of salinity [control (60), 80, 120, $160 \mathrm{Mm}$ $\mathrm{NaCl}$, on five genotypes of quinoa in case of germination and grain weight/plant. The serial number, name and source of the genotypes materials are presented in Table 1.

Table (1): The serial number, name and source of the genotypes materials.

\begin{tabular}{|c|c|c|}
\hline No. of entry & Genotype name & Genotype Source \\
\hline 1 & M-28 & Denmark \\
2 & Q-37 & Chile \\
3 & S-10 & Denmark \\
4 & Regeolone-3 & Chile \\
5 & Line -6 & Denmark \\
\hline
\end{tabular}


The experimental design was completely randomized design (CRD) in split -devoted plots with three replications. The main plots were devoted to four salinity levels [control (60), 80, 120 and $160 \mathrm{Mm} \mathrm{NaCl}$, while, the five genotypes were assigned to the sub plots. Day. Plastic pots of $15 \mathrm{~cm}$ diameter and $16 \mathrm{~cm}$ depth were filled with $3.00 \mathrm{~kg}$ mixture of 1: 1 sand: clay. Planting dates were on $13^{\text {th }}$ and $26^{\text {th }}$ November at 2016/ 2017 and 2017/2018 seasons. After 4 days seedlings were thinned at rate of 25 seedlings/ genotype. Phosphoric acid $\left(\mathrm{H}_{2} \mathrm{PO}_{5}\right.$, $85 \%$ ) at rate of $1 \mathrm{~cm} / \mathrm{L}$ and NPK (20: 20: 20 ) at rate of $1 \mathrm{~g} / \mathrm{L}$ were added. The salinity levels treatments were applied after 30 days from planting until three months later. The soil salinity was $4.46 \mathrm{dsm}^{-1}$. Harvesting date was after 110 days from sowing date.

\section{Germination}

The experiment was conducted in vitro. Grains were surface-sterilized for $20 \mathrm{~min}$ in $20 \%$ sodium hypochlorite, rinsed and soaked for $1 \mathrm{~h}$ in distilled water. Germination carried out at $25 \pm 2{ }^{\circ} \mathrm{C}$ under dark conditions, the germination of quinoa by using saline water with filter paper in Petri dishes. Saline water developed by using $\mathrm{NaCl} 1.35 \mathrm{~g}$, $3.51 \mathrm{~g}, 6.03 \mathrm{~g}$, in $1 \mathrm{~L}$ of distilled water to make $60,80,120$ and $160 \mathrm{Mm}$ treatments. Quinoa grains (25) were sown in the Petri dishes with 3 replicates. Germination checked regularly 3 days after sowing date.

\section{Germination parameters}

The germination parameters were calculated for all germination experiments during the first 20 days of the germination test, which consisted of:

\section{Germination Rate (GR)}

Defined according to Barlett (1973) as following:

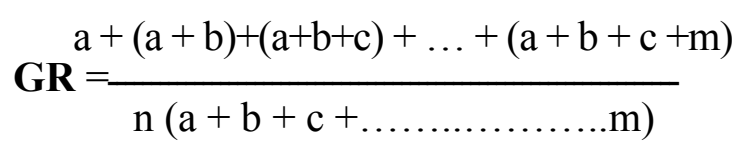

Where:

(a) Number of seedlings emerged at the first count. (b) Number of seedlings emerged at the second count.

(c) Number of seedlings emerged at the $3^{\text {rd }}$ count.

(m) Number of seedlings emerged at the final count.

(n) Number of counts.

\section{Yield and yield components}

At harvest date (110 days from sowing) a random sample of ten guarded plants were taken from each pot to measure the following characters:

\section{Grain weight (g/plant)}

It was determined as an average of grain weight on plant basis.

\section{Harvest index (\%)}

It was calculated as a percentage of grain weight (g/plant) divided to plant fresh weight $(\mathrm{g})$.

\section{0-grain weight $(\mathrm{g})$}

It was determined as an average of 1000grain weight from per plant.

\section{Statistical Analysis}

Results were statistical analyzed with analysis of variance (ANOVA) procedure using the General Linear Models (GLMs) procedures using SAS (SAS, 2004). Differences between means were compared by using Duncan's multiple ranged tests (Duncan, 1955).

\section{RESULTS AND DISCUSSION}

Germination rate $(\mathrm{GR} \%)$ and germination percentage (GP \%)

Resuls in Table 2 illustrate that there were significant differences between salinity levels on germination rate and germination percentage.

They were decreased, when salt concentration increased. The highest values of germination rate $(61.22,80.80 \%)$ were obtained by $60 \mathrm{Mm}$ treatment at both seasons. While, the lowest germination rate 
Table (2): Effect of salinity levels on Quinoa germination rate (GR\%) and germination percentage (GP\%) at 2016-2017 and 2017-2018 seasons.

\begin{tabular}{c|cc|cc}
\hline \multirow{2}{*}{$\begin{array}{c}\text { Salinity NaCl } \\
(\mathbf{M m})\end{array}$} & \multicolumn{2}{|c|}{ Germination rate (GR (\%) } & \multicolumn{2}{c}{ Germination percentage (GP) (\%) } \\
\cline { 2 - 5 } & $\mathbf{2 0 1 6 / 2 0 1 7}$ & $\mathbf{2 0 1 7 / 2 0 1 8}$ & $\mathbf{2 0 1 6 / 2 0 1 7}$ & $\mathbf{2 0 1 7 / 2 0 1 8}$ \\
\hline $\mathbf{6 0}$ & $61.22^{\mathrm{a}}$ & $80.88^{\mathrm{a}}$ & $71.81^{\mathrm{a}}$ & $78.40^{\mathrm{a}}$ \\
$\mathbf{8 0}$ & $58.86^{\mathrm{ab}}$ & $78.28^{\mathrm{a}}$ & $58.86^{\mathrm{ab}}$ & $70.51^{\mathrm{ab}}$ \\
$\mathbf{1 2 0}$ & $55.90^{\mathrm{ab}}$ & $74.28^{\mathrm{ab}}$ & $56.24^{\mathrm{bc}}$ & $59.56^{\mathrm{bc}}$ \\
$\mathbf{1 6 0}$ & $52.89^{\mathrm{b}}$ & $69.83^{\mathrm{b}}$ & $48.36^{\mathrm{c}}$ & $55.60^{\mathrm{c}}$ \\
\hline
\end{tabular}

*Means followed by the same letters within the same column are not significantly different according to Duncan's multiple range test at $(\mathrm{P} \leq 0.05)$

$(52.89,69.83 \%)$ were found with $160 \mathrm{Mm}$ treatment at the both seasons $(2017$ and 2018). This may refers to the osmotic potential may be the reason for germination delay. The reduction of radicle growth under salt stress conditions may due to the diminution in the turgor of radicle cells. These results are in agreement with those found by Panuccio et al. (2014).

The highest values of GP $(71.81,78.4 \%)$ were obtained by $60 \mathrm{Mm}$ treatment at both seasons While, the lowest $(48.36,55.60 \%)$ were achieved by irrigated water with salinity level of $160 \mathrm{Mm}$ at the both seasons. These results are in harmony with those detected by Begum et al. (2010). Also, Arshadullah et al. (2016) found that the germination percentage ranged from 90 to $100 \%$ by $14 \mathrm{dSm}^{-1}$ and drastically reduced to $65 \%$ at $16 \mathrm{dSm}^{-1}$. There were significant differences between genotypes on germination rate and germination percentage (Table 3). The highest values of GR $(71.72,87.9 \%)$ were found with the M28 genotype at the first and second seasons. While, the lowest $(44.08,60.23 \%)$ were achieved with the Line- 6 genotype at the both seasons. These results were in coordinate with those reported by Tan and kcay, 2017. In addition, Sanghera et al. (2016) on sugar beet genotypes revealed that the germination rates (\%) varied from $60 \%$ (Calixta) to $89.67 \%$ (Cauvery).
Regjnding to germination percentage of quinoa seeds. The maximum value of GP79.8 \% was obtained by the M-28 genotype at first season, but each of M-28 and Q-37 genotypes gave valued $(87.07 \%$ and $78.90 \%$ ) at the second season. However, the minimum value was scored by the Line-6genotype at the both seasons $(45.74,50.33 \%)$, respectively. These results coincided with those obtained by Sourour et al. (2014) on wheat, whereas they showed that the increase in $\mathrm{NaCl}$ concentrations decreased germination percentage.

Also, the results in Table 4 indicate a significant interaction differences between salinity and genotypes on germination rate and germination percentage. The highest GR and GP\% (77.27, 65.21 and 93.76, $96.33 \%$, in respectively) were obtained by the M-28 genotype under $60 \mathrm{Mm}$ treatment at both seasons (2016/2017 and 2017/ 2018). While, the lowest GR and GP\% (40.35, 55.2\% and 34.73, 35.04\%, in respectively) were obtained by the Line- 6 genotype under $160 \mathrm{Mm}$ treatment at the both seasons.

Results in Table 5 illustrate that there were significant differences between salinity levels on grain weight, harvest index and 1000-grain weight. The highest values of the harvest index $(33.0 \& 42.0 \%$ and $33.0 \& 41 \%$ ), respectively were attained at the $60,80 \mathrm{Mm}$ treatments at both 
SINAI Journal of Applied Sciences (ISSN: 2314-6079) Vol. (8) Is. (2), Aug. 2019

Table (3): Germination rate (GR \%) and germination percentage (GP \%) for the five genotypes at 2016-2017 and 2017-2018 seasons.

\begin{tabular}{c|cccc}
\hline \multirow{2}{*}{ Genotype } & \multicolumn{2}{|c}{ Germination rate (GR) (\%) } & \multicolumn{2}{c}{ Germination percentage (GP) (\%) } \\
\cline { 2 - 5 } & $\mathbf{2 0 1 6 / 2 0 1 7}$ & $\mathbf{2 0 1 7 / 2 0 1 8}$ & $\mathbf{2 0 1 6 / 2 0 1 7}$ & $\mathbf{2 0 1 7 / 2 0 1 8}$ \\
\hline Q-37 & $61.71^{\mathrm{b}}$ & $82.01^{\mathrm{b}}$ & $69.44^{\mathrm{b}}$ & $78.90^{\mathrm{a}}$ \\
Regeolona-3 & $56.75^{\mathrm{c}}$ & $76.82^{\mathrm{c}}$ & $55.15^{\mathrm{c}}$ & $61.98^{\mathrm{b}}$ \\
S-10 & $51.83^{\mathrm{d}}$ & $72.05^{\mathrm{d}}$ & $48.50^{\mathrm{cd}}$ & $51.81^{\mathrm{c}}$ \\
M-28 & $71.72^{\mathrm{a}}$ & $87.97^{\mathrm{a}}$ & $79.81^{\mathrm{a}}$ & $87.07^{\mathrm{a}}$ \\
Line-6 & $44.08^{\mathrm{e}}$ & $60.23^{\mathrm{e}}$ & $45.74^{\mathrm{d}}$ & $50.33^{\mathrm{c}}$
\end{tabular}

*Means followed by the same letters within the same column are not significantly different according to Duncan's multiple range test at $(\mathrm{P} \leq 0.05)$

Table (4): Effect of interaction between salinity levels and genotypes on germination rate (GR $\%$ ) and germination percentage (GP \%) at 2016-2017 and 2017-2018 seasons.

\begin{tabular}{|c|c|c|c|c|c|}
\hline \multirow{2}{*}{$\begin{array}{c}\text { Salinity } \\
\text { NaCl (Mm) }\end{array}$} & \multirow{2}{*}{ Genotype } & \multicolumn{2}{|c|}{ Germination rate (GR) (\%) } & \multicolumn{2}{|c|}{$\begin{array}{c}\text { Germination percentage (GP } \\
(\%)\end{array}$} \\
\hline & & $2016 / 2017$ & $2017 / 2018$ & $2016 / 2017$ & $2017 / 2018$ \\
\hline 60 & $\begin{array}{c}\text { Q-37 } \\
\text { Regeolona-3 } \\
\text { S-10 } \\
\text { M-28 } \\
\text { Line-6 }\end{array}$ & $\begin{array}{l}65.12^{\mathrm{d}} \\
61.21^{\mathrm{f}} \\
55.27^{\mathrm{i}} \\
77.27^{\mathrm{a}} \\
47.22^{\mathrm{n}}\end{array}$ & $\begin{array}{l}87.29^{\mathrm{c}} \\
82.33^{\mathrm{e}} \\
77.40^{\mathrm{h}} \\
92.18^{\mathrm{a}} \\
65.21^{\mathrm{m}}\end{array}$ & $\begin{array}{l}85.80^{\mathrm{b}} \\
66.13^{\mathrm{e}} \\
58.06^{\mathrm{f}} \\
93.76^{\mathrm{a}} \\
55.30^{\mathrm{fg}}\end{array}$ & $\begin{array}{c}91.16^{\mathrm{ab}} \\
76.46^{\mathrm{cd}} \\
67.00^{\mathrm{def}} \\
96.33^{\mathrm{a}} \\
61.06^{\mathrm{fg}}\end{array}$ \\
\hline 80 & $\begin{array}{c}\text { Q-37 } \\
\text { Regeolona-3 } \\
\text { S-10 } \\
\text { M-28 } \\
\text { Line-6 }\end{array}$ & $\begin{array}{l}63.30^{\mathrm{e}} \\
58.21^{\mathrm{h}} \\
53.32^{\mathrm{j}} \\
74.19^{\mathrm{b}} \\
45.28^{\mathrm{o}}\end{array}$ & $\begin{array}{l}85.21^{\mathrm{d}} \\
79.34^{\mathrm{g}} \\
74.35^{\mathrm{j}} \\
90.21^{\mathrm{b}} \\
62.28^{\mathrm{n}}\end{array}$ & $\begin{array}{l}71.13^{\mathrm{d}} \\
57.90^{\mathrm{f}} \\
52.33^{\mathrm{gh}} \\
82.83^{\mathrm{b}} \\
48.33^{\mathrm{hij}}\end{array}$ & $\begin{array}{l}84.36^{\text {bc }} \\
64.60^{\text {ef }} \\
58.10^{\text {fgh }} \\
94.00^{\mathrm{ab}} \\
51.50^{\text {ghi }}\end{array}$ \\
\hline 120 & $\begin{array}{c}\text { Q-37 } \\
\text { Regeolona-3 } \\
\text { S-10 } \\
\text { M-28 } \\
\text { Line-6 }\end{array}$ & $\begin{array}{l}60.21^{\mathrm{g}} \\
55.21^{\mathrm{i}} \\
50.41^{\mathrm{L}} \\
70.21^{\mathrm{c}} \\
43.47^{\mathrm{p}}\end{array}$ & $\begin{array}{l}80.33^{\mathrm{f}} \\
75.30^{\mathrm{i}} \\
70.35^{\mathrm{k}} \\
87.26^{\mathrm{c}} \\
58.16^{\mathrm{o}}\end{array}$ & $\begin{array}{l}64.76^{\mathrm{e}} \\
51.06^{\mathrm{hi}} \\
47.43^{\mathrm{ij}} \\
76.10^{\mathrm{c}} \\
41.8^{\mathrm{kl}}\end{array}$ & $\begin{array}{c}74.50 \mathrm{cde} \\
56.13 \mathrm{fgh} \\
47.36 \mathrm{hi} \\
84.76 \mathrm{bc} \\
47.10 \mathrm{hi}\end{array}$ \\
\hline 160 & $\begin{array}{c}\text { Q-37 } \\
\text { Regeolona-3 } \\
\text { S-10 } \\
\text { M-28 } \\
\text { Line-6 }\end{array}$ & $\begin{array}{l}58.23^{\mathrm{h}} \\
52.36^{\mathrm{k}} \\
48.31^{\mathrm{m}} \\
65.22^{\mathrm{d}} \\
40.35^{\mathrm{q}}\end{array}$ & $\begin{array}{l}75.21^{\mathrm{i}} \\
70.31^{\mathrm{k}} \\
66.11^{\mathrm{L}} \\
82.22^{\mathrm{e}} \\
55.27^{\mathrm{p}}\end{array}$ & $\begin{array}{l}56.06^{\mathrm{fg}} \\
45.50^{\mathrm{jk}} \\
38.96^{1} \\
66.56^{\mathrm{e}} \\
34.73^{\mathrm{m}}\end{array}$ & $\begin{array}{c}65.56^{\text {def }} \\
50.73^{\text {ghi }} \\
41.40^{\mathrm{ij}} \\
73.20^{\mathrm{de}} \\
35.04^{\mathrm{j}}\end{array}$ \\
\hline
\end{tabular}

*Means followed by the same letters within the same column are not significantly different according to Duncan's multiple range test at $(\mathrm{P} \leq 0.05)$ 
Table (5): Effect of salinity levels on grain weight, harvest index and 1000- Grain weight at 2016-2017 and 2017-2018 seasons.

\begin{tabular}{|c|c|c|c|c|c|c|}
\hline \multirow{2}{*}{$\begin{array}{c}\text { Salinity } \\
\text { NaCl (Mm) }\end{array}$} & \multicolumn{2}{|c|}{ Grain weight ( g/plant) } & \multicolumn{2}{|c|}{ Harvest Index (\%) } & \multicolumn{2}{|c|}{ 1000-Grain weight(g) } \\
\hline & $2016 / 2017$ & $2017 / 2018$ & $2016 / 2017$ & $2017 / 2018$ & $2016 / 2017$ & $2017 / 2018$ \\
\hline 60 & $6.69^{\mathrm{a}}$ & $9.43^{\mathrm{a}}$ & $33.00^{\mathrm{a}}$ & $42.00^{\mathrm{a}}$ & $1.68^{\mathrm{a}}$ & $1.87^{\mathrm{a}}$ \\
\hline 80 & $6.29^{\mathrm{ab}}$ & $8.75^{\mathrm{a}}$ & $\begin{array}{l}33.00^{\mathrm{a}} \\
30.00^{\mathrm{a}}\end{array}$ & $\begin{array}{l}41.00^{\mathrm{a}} \\
35.00^{\mathrm{b}}\end{array}$ & $1.55^{\mathrm{ab}}$ & $1.76^{\mathrm{a}}$ \\
\hline 120 & $4.74^{\mathrm{b}}$ & $6.49^{\mathrm{b}}$ & $21.00^{\mathrm{b}}$ & $30.00^{\mathrm{c}}$ & $1.48^{\mathrm{b}}$ & $1.54^{\mathrm{b}}$ \\
\hline 160 & $2.87^{\mathrm{c}}$ & $4.64^{\mathrm{c}}$ & & & $1.00^{\mathrm{c}}$ & $1.11^{\mathrm{c}}$ \\
\hline
\end{tabular}

*Means followed by the same letters within the same column are not significantly different according to Duncan's multiple range test at $(\mathrm{P} \leq 0.05)$

seasons. When, the minimum harvest index was found by $160 \mathrm{Mm} \mathrm{NaCl}$ at both seasons $(21.0,30.0 \%)$. These results were in agreement with those detected by Hirich et al. (2014).

As for grain weight and 1000-grain weight, the result in Table 5 showed that maximum value of grain weight and 1000grains weight were $(6.69,9.43 \mathrm{~g}$ and 1.68 , $1.87 \mathrm{~g}$, respectively) with $60 \mathrm{Mm}$ treatment at both seasons. Followed in ding the salts in Table 5 by $(8.75,1.76 \mathrm{~g})$ which obtained by $80 \mathrm{Mm}$ treatment at the second season. While, the minimum value was obtained from $160 \mathrm{Mm}$ treatment at the both seasons (2.87, 4.64 and $1.00,1.11 \mathrm{~g})$, respectively. These results were in the same line with those stated by Algosaibi et al. (2015). There were significant differences between genotypes on grain weight, harvest index and 1000-grain weight (Table 6). The highest grain weight (g/plant) and 1000-grains weight (8.00, $11.02 \mathrm{~g}$ and $1.88,2.00 \mathrm{~g}$, respectively) were obtained by M-28 genotype at both seasons. When, the lowest values 2.63, 4.83 $\mathrm{g}$ and $1.22,1.62 \mathrm{~g}$, respectively were found by Line- 6 genotype at both seasons. As for harvest Index, the result in Table 6 showed that maximum harvest index value was obtained by $32.0 \%$ with M-28 genotype followed by $30.0 \%$ with Q-37 genotype at the first season, but that maximum value obtained by the M-28 genotype was $42.0 \%$ at second season. While, the lowest harvest index scolded $(29.0 \& 34.0 \%),(29.0 \&$ $0.36 \%)$ and $(28.0 \& 36.0 \%)$, respectively were found by the Regeolona-3, S-10 and Line-6 genotypes at both seasons. These results were alleged with the previous results, which obtained by Miranda et al. (2013) and they were showed that in the case of 'Regalona Baer' and 'Villarrica' genotypes a significant increase in grain yield (4.2 and $5.1 \mathrm{t} \mathrm{ha-}^{1}$, respectively) and 1000 grain weight $(3.08 \pm 0.08$ and $3.29 \pm$ $0.08 \mathrm{~g}$, respectively).

Results in Table 7 indicat a significant differences interaction between salinity levels and genotypes quinoa on grain weight, harvest index and 1000-grain weight. The highest grain weight (g/plant) $(10.35,13.29 \mathrm{~g})$, harvest index $(37.0,47.0 \%)$ and 1000- grain weight $(2.80,3.84)$, respectively were obtained by $\mathrm{M}-28$ genotype with $60 \mathrm{Mm}$ treatment $(60 \mathrm{Mm})$ at both seasons, while the lowest $(1.7,2.63 \mathrm{~g})$, $(21.0,28.0 \%)$ and $(0.91,1.03 \mathrm{~g})$, respectively for Line-6 genotype with160 Mm treatment through both seasons. 
SINAI Journal of Applied Sciences (ISSN: 2314-6079) Vol. (8) Is. (2), Aug. 2019

Table (6): Differences among quinoa genotypes in concern of grain weight, harvest index and 1000- Grains weight at 2016/2017 and 2017/2018 seasons.

\begin{tabular}{|c|c|c|c|c|c|c|c|}
\hline \multirow{2}{*}{$\begin{array}{c}\text { Salinity } \\
\mathrm{NaCl} \\
(\mathrm{Mm})\end{array}$} & \multirow{2}{*}{ Genotypes } & \multicolumn{2}{|c|}{ Grain weight ( g/plant) } & \multicolumn{2}{|c|}{ Harvest Index (\%) } & \multicolumn{2}{|c|}{ 1000- Grain weight(g) } \\
\hline & & $2016 / 2017$ & $2017 / 2018$ & $2016 / 2017$ & $2017 / 2018$ & $2016 / 2017$ & $2017 / 2018$ \\
\hline \multirow{5}{*}{60} & Q-37 & $8.37^{\mathrm{b}}$ & $10.51^{\mathrm{b}}$ & $36.00^{\mathrm{abc}}$ & $39.00^{\mathrm{cd}}$ & $2.37^{\mathrm{b}}$ & $2.73^{\mathrm{c}}$ \\
\hline & Regeolona-3 & $4.75^{\mathrm{f}}$ & $7.45^{\mathrm{e}}$ & $31.00^{\mathrm{c}-\mathrm{e}}$ & $38.00^{\text {de }}$ & $2.09^{\text {cde }}$ & $2.17^{\mathrm{efg}}$ \\
\hline & S-10 & $6.65^{\mathrm{d}}$ & $9.29^{c}$ & $34.00^{\mathrm{cbd}}$ & $41.00^{\mathrm{cbd}}$ & $2.15^{\mathrm{cbd}}$ & $2.40^{\mathrm{de}}$ \\
\hline & M28 & $10.35^{\mathrm{a}}$ & $13.29^{\mathrm{a}}$ & $37.00^{\mathrm{ab}}$ & $47.00^{\mathrm{a}}$ & $2.80^{\mathrm{a}}$ & $3.84^{\mathrm{a}}$ \\
\hline & Line-6 & $3.33^{\mathrm{h}}$ & $6.62 \mathrm{f}$ & $28.00^{\mathrm{def}}$ & $42.00^{\mathrm{cb}}$ & $1.73^{\mathrm{fg}}$ & $2.13^{\mathrm{fg}}$ \\
\hline \multirow{5}{*}{80} & Q-37 & $8.10^{b}$ & $9.48^{\mathrm{c}}$ & $36.00^{\mathrm{abc}}$ & $40.00^{\text {cbd }}$ & $2.25^{\mathrm{cbd}}$ & $2.48^{\mathrm{d}}$ \\
\hline & Regeolona-3 & $4.13^{\mathrm{g}}$ & $6.49^{\mathrm{f}}$ & $23.00^{\mathrm{fg}}$ & $39.00^{\mathrm{cd}}$ & $1.86^{\mathrm{ef}}$ & $2.11^{\mathrm{fg}}$ \\
\hline & S-10 & $6.05^{\mathrm{e}}$ & $8.45^{\mathrm{d}}$ & $38.00^{\mathrm{d}}$ & $41.00^{\mathrm{cbd}}$ & $2.08^{\text {cde }}$ & $2.30^{\text {def }}$ \\
\hline & M-28 & $10.07^{\mathrm{a}}$ & $13.13^{\mathrm{a}}$ & $42.00 \mathrm{a}$ & $43.00^{\mathrm{b}}$ & $2.34^{\mathrm{cb}}$ & $3.38^{\mathrm{b}}$ \\
\hline & Line-6 & $3.10^{\mathrm{hi}}$ & $6.20^{\mathrm{f}}$ & $23.00^{\mathrm{fg}}$ & $40.00^{\mathrm{d}}$ & $1.23^{\mathrm{ij}}$ & $2.03^{\mathrm{g}}$ \\
\hline \multirow{5}{*}{120} & Q-37 & $6.26^{\text {ed }}$ & $7.30^{\mathrm{e}}$ & $32.00^{\mathrm{c}-\mathrm{e}}$ & $35.00^{\mathrm{ef}}$ & $2.10^{\mathrm{c}-\mathrm{e}}$ & $2.13^{\mathrm{fg}}$ \\
\hline & Regeolona-3 & $4.32^{\mathrm{fg}}$ & $4.43^{h}$ & $37.00^{\mathrm{ab}}$ & $0.30^{\text {ghi }}$ & $1.42^{\mathrm{hi}}$ & $1.38^{\mathrm{i}}$ \\
\hline & S-10 & $3.46^{\mathrm{h}}$ & $6.52^{\mathrm{f}}$ & $22.00^{\mathrm{fg}}$ & $0.35^{\text {ef }}$ & $2.13^{\mathrm{c}-\mathrm{e}}$ & $1.74^{\mathrm{h}}$ \\
\hline & M-28 & $7.28^{\mathrm{c}}$ & $10.38^{\mathrm{b}}$ & $31.00^{\mathrm{c}-\mathrm{e}}$ & $42.00^{\mathrm{cb}}$ & $2.24^{\mathrm{bcd}}$ & $2.35^{\mathrm{def}}$ \\
\hline & Line-6 & $2.40^{\mathrm{j}}$ & $3.85^{\mathrm{ij}}$ & $22.00^{\mathrm{fg}}$ & $31.00^{\text {ghi }}$ & $1.03^{\mathrm{jk}}$ & $1.31^{\mathrm{i}}$ \\
\hline \multirow{5}{*}{160} & Q-37 & $3.25^{\mathrm{h}}$ & $5.53^{\mathrm{g}}$ & $25.00^{\text {efg }}$ & $32.00^{\mathrm{fgh}}$ & $1.52^{\mathrm{gh}}$ & $1.28 \mathrm{i}$ \\
\hline & Regeolona-3 & $2.44^{\mathrm{j}}$ & $3.47^{\mathrm{j}}$ & $21.00^{\mathrm{g}}$ & $28.00^{\mathrm{hi}}$ & $1.14^{\mathrm{jk}}$ & $1.13^{\mathrm{ij}}$ \\
\hline & S-10 & $2.64^{\mathrm{ij}}$ & $4.28^{\text {ih }}$ & $20.00^{\mathrm{g}}$ & $28.00^{\mathrm{hi}}$ & $1.12^{\mathrm{jk}}$ & $1.16^{\mathrm{ij}}$ \\
\hline & M-28 & $4.30^{\mathrm{fg}}$ & $7.30^{\mathrm{e}}$ & $26.00^{\text {efg }}$ & $34.00^{\text {fgh }}$ & $2.04^{\mathrm{de}}$ & $2.01^{\mathrm{g}}$ \\
\hline & Line-6 & $1.71^{\mathrm{k}}$ & $2.63^{\mathrm{k}}$ & $13.00^{\mathrm{h}}$ & $27.00^{\mathrm{i}}$ & $0.91^{\mathrm{k}}$ & $1.03^{\mathrm{j}}$ \\
\hline
\end{tabular}

Table (7): Effect of interaction between salinity levels and genotypes on grain weight, harvest index and1000- Grains weight at 2016-2017 and 2017-2018 seasons.

\begin{tabular}{|c|c|c|c|c|c|c|}
\hline \multirow{2}{*}{ Genotypes } & \multicolumn{2}{|c|}{ Grain weight ( g/plant) } & \multicolumn{2}{|c|}{ Harvest Index (\%) } & \multicolumn{2}{|c|}{ 1000-Grain weight $(g)$} \\
\hline & $2016 / 2017$ & $2017 / 2018$ & $2016 / 2017$ & $2017 / 2018$ & $2016 / 2017$ & $2017 / 2018$ \\
\hline $\begin{array}{c}\text { Q-37 } \\
\text { Regeolona-3 S-10 } \\
\text { M-28 } \\
\text { Line-6 }\end{array}$ & $\begin{array}{c}6.50^{\mathrm{b}} \\
3.91^{\mathrm{cd}} \\
6.50^{\mathrm{c}} \\
8.00^{\mathrm{a}} \\
2.63^{\mathrm{d}}\end{array}$ & $\begin{array}{c}8.20^{\mathrm{b}} \\
5.46^{\mathrm{c}} \\
7.13^{\mathrm{b}} \\
11.02^{\mathrm{a}} \\
4.83^{\mathrm{c}}\end{array}$ & $\begin{array}{l}30.00^{\mathrm{a}} \\
29.00^{\mathrm{b}} \\
29.00^{\mathrm{b}} \\
32.00^{\mathrm{a}} \\
28.00^{\mathrm{b}}\end{array}$ & $\begin{array}{l}36.00^{\mathrm{b}} \\
34.00^{\mathrm{b}} \\
36.00^{\mathrm{b}} \\
42.00^{\mathrm{a}} \\
36.00^{\mathrm{b}}\end{array}$ & $\begin{array}{c}1.77^{\mathrm{ab}} \\
1.62^{\mathrm{c}} \\
1.72^{\mathrm{bc}} \\
1.88^{\mathrm{a}} \\
1.22^{\mathrm{d}}\end{array}$ & $\begin{array}{l}1.16^{\mathrm{b}} \\
1.70^{\mathrm{bc}} \\
1.90^{\mathrm{bc}} \\
2.00^{\mathrm{a}} \\
1.62^{\mathrm{c}}\end{array}$ \\
\hline
\end{tabular}

*Means followed by the same letters within the same column are not significantly different according to Duncan's multiple range test at $(\mathrm{P} \leq 0.05)$.

\section{REFERENCES}

Algosaibi, A.M.; El-Garawany, M.M.; Badran, A.E.; A.M. Almadini (2015). Effect of irrigation water salinity on the growth of quinoa plant. J. Agric. Sci., 7 (8): 205.

Arshadullah, M.; Suhaib, M.; Usama, M.B.; Mahmood, I.A. and Hyder, S.I. (2016). Effect of salinity on growth of
Chenopodium quinoa Willd. J. Res. Agaric, Forestry, 11(3): 21-24.

Barlett, M.S (1973). Some samples of statistical method of research in agriculture and applied biology. J. Roy. Soc., 4: 2.

Bascunan-Godo, L.; Reguera, M.; AbdelTawab, Y.M. and Blumwald, E. (2015). Water deficit stress-induced changes in carbon and nitrogenpartitioning in 
Chenopodium quinoa Willaad. Planta, 1: 13.

Begum, F.; Ahmed, I.M.; Nessa, A. and Sultana, W. (2010). The effect of salinity on seed quality of wheat. J. Bangladesh Argic. Univ., 8 (1): 19-22.

Duncan, B.D. (1955). Multiple ranges and multiple F test. Biomet., 11: 1-42.

Eisa, S.; Hussin, S.; Geissler, N. and Koyro, H.W. (2012). Effect of $\mathrm{NaCl}$ salinity on water relations, photosynthesis and chemical composition of quinoa (Chenopodium quinoa Willd.) as a potential cash crop halophyte. AJCS. 6 (2): 357-368. (Aust. J. Crop Sci.).

FAO (2011). Quinoa: an ancient crop to contribute to world food security. Food and Agric. Organization of the United Nations.

Hirich, A.; Jelloul, A.; Choukr-Allah, R. and Jacobsen, S.E. (2014). Saline Water Irrigation of Quinoa and Chickpea: seedling rate, stomata conductance and yield responses. J. Agro. Crop. Sic., 200: 378-389.

Miranda, M.; Vega-Gálvez, A.; Martínez, E.A.; López, J.; Marín, R.; Aranda, M. and Fuentes, F. (2013). Influence of contrasting environments on seed composition of two quinoa genotypes: nutritional and functional properties. J. Agro. Res., 73 (2): 108-116.

Panuccio, M.R.; S.E. Jacobsen; S.S. Akhta; A. Muscolo (2014). Effect of saline water irrigation on seed germination and early seedling growth of the halophyte quinoa. J. Agri. Sci., 1-18.

Sanghera, G.S.; Singh, R.P.; Kashyap, L.; Tyagi, V. and Sharma, B. (2016). Evaluation of sugar beet genotypes (Beta Vulgaris L.) for root yield and quality traits under subtropical conditions. Punjab. Agric. Univ. Regional. Res. Station, Kapurthala. J. Krishi. Vigyan., 5 (1): 67-73.

SAS (2004). SAS/STAT User's Guide. SAS Institute Inc., Cary, N.C.

Stevens, M.R.; Coleman, C.E.; Parkinson, S.E.; Maughan, P.J.; Zhang, H.B.; Balzotti, M.R.; Kooyman, D.L.; Arumuganathan, K.; Bonifacio, A.; Fairbanks, D.J.; Jellen, E.N. and Stevens, J.J. (2006). Construction of a quinoa (Chenopodium quinoa Willd.) BAC library and its use in identifying genes encoding seed storage proteins. Theory. Appl Genet., 112: 1593-1600.

Sourour, A.; Neila, R.; Zoubeir, C.; Saddreddine, B.; Feker, K.; Themir, B.; Mayada, M. and Mongi, B.Y. (2014). Effect of salt stress (sodium chloride) on germination and seedling growth of durum wheat (Triticum durum Desf.) genotypes. Int. J. Biodivers. Conserv., 6 (4): 320-325.

Tan, M. and Kcay, E.A. (2017). Temperature and salinity effects on germination of some quinoa (chenopodium quinoa Willd.) cultivars. Proc. of $89^{\text {th }}$ the IRES Int. Conf., Helsinki, Finland: 3-5. 


$$
\text { الملخص العربي }
$$

\section{أداء بعض التراكيب الوراثية لنبات الكينوا تحت مستويات مختلفة من الملوحة

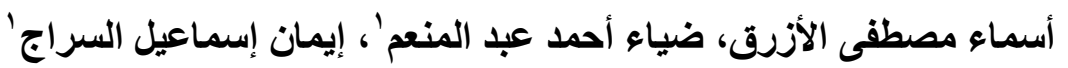 \\ 1 ـ قسم الإنتاج النباتي، كلية العلوم الزراعية البيئية، جامعة العريش، مصر.}

أجريت تجربة بالصوبة الزراعية بالمزرعة التجريبية لكليه العلوم الزراعية البيئية، جامعة العريش، محافظة شمال

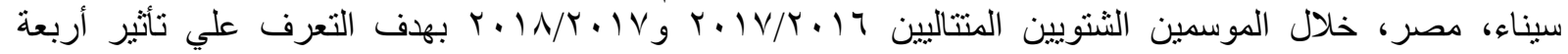

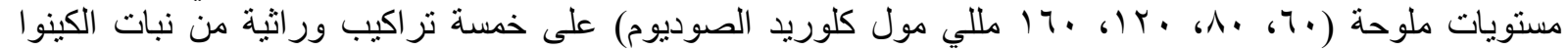
في حالة الإنبات ووزن الحبوب للنبات، حيث تم إستخدام التصميم (M28, Q37, S10, Regeolone3, Line 6)

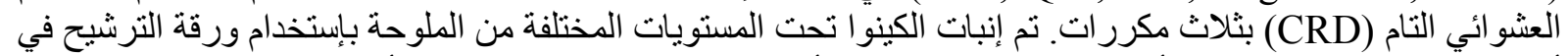

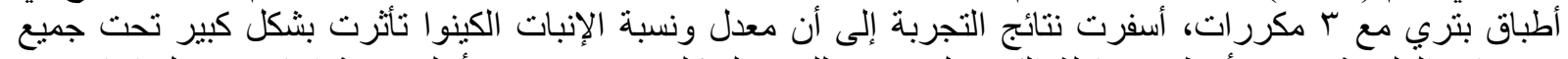

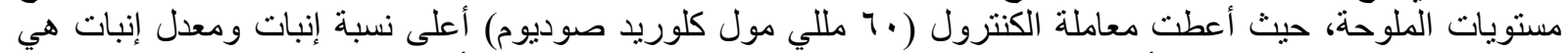

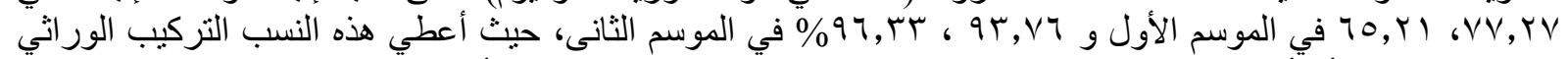

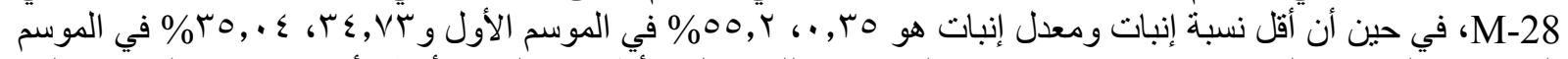

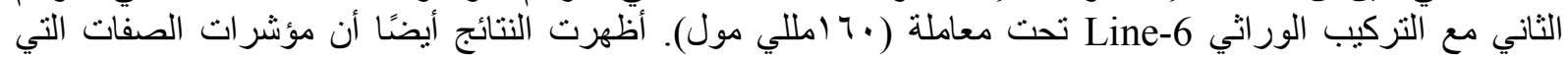

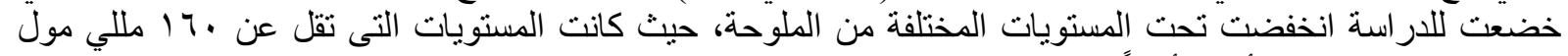

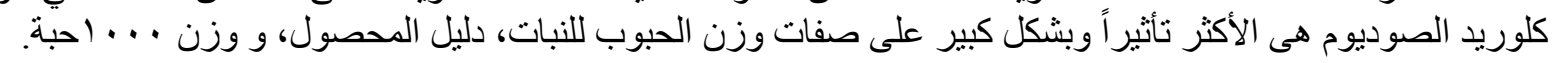

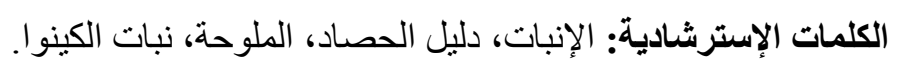


